\title{
A Multicenter Observational Prospective Study of the Preferred Inhaler After a Switch of Therapy in Asthma Patients
}

Sören Wille $\cdot$ Holger von Fircks

To view enhanced content go to www.pulmonarytherapy-open.com

Received: August 28, 2015 / Published online: November 2, 2015

(C) The Author(s) 2015. This article is published with open access at Springerlink.com

\section{ABSTRACT}

Introduction: Poor compliance and incorrect handling of inhaler devices is common among patients with asthma using traditional dry powder inhalers, and patient preference and perception of ease of handling are important. The aim of this study was to determine the proportion of patients preferring the recently introduced Novopulmon ${ }^{\circledR}$ Novolizer $^{\circledR}$ device (Meda, Sweden) compared with their previous inhaler device.

Methods: An observational study was conducted among 258 asthma patients in Sweden. Patients aged $\geq 6$ years, receiving maintenance treatment with inhaled corticosteroids, and requiring a switch to another inhaler were included. Patients

Electronic supplementary material The online version of this article (doi:10.1007/s41030-015-0006-7) contains supplementary material, which is available to authorized users.

S. Wille $(\bowtie)$

Barnmottagningen, Helsingborg Hospital,

Helsingborg, Sweden

e-mail: soren.wille@skane.se

H. von Fircks $(\square)$

Meda AB, Solna, Sweden

e-mail: hvf@meda.se completed a questionnaire regarding the preference, appearance, handling, instructions for use, dose control, and global perception of the inhaler upon switching the device and again after 2 months' use of the new device.

Results: Patients were aged from 6 to 85 years. Almost all patients (98\%) were switched to the Novolizer inhaler. The majority of patients preferred Novolizer versus their previous inhaler: $92.4 \%$ [95\% confidence interval (CI) 86.0-96.5\%] in the 6-12 years age group, 90.9\% (95\% CI 70.8-98.9\%) in the 13-17 years group, and $83.3 \%$ (95\% CI 74.4-90.2\%) among those aged $\geq 18$ years. The results from 10 questions related to the inhalation process and device handling demonstrated that Novopulmon Novolizer was evaluated by patients as superior compared with Turbuhaler $^{\circledR}$ (AstraZeneca), Diskus $^{\circledR} \quad$ (GlaxoSmithKline) and Easyhaler ${ }^{\circledR}$ (Orion); $P<0.022$ for all comparisons.

Conclusion: Novopulmon Novolizer was determined by patients to be the preferred device compared with previously used devices, and was the patients' preference for continued use. In particular, the Novolizer's audible, visual and sensory feedback mechanisms (that identify whether the correct dose is loaded and 
administered) and its general ease of use were highly appreciated by asthma patients. Together this makes the Novopulmon Novolizer an ideal first choice inhaler for treatment of asthma, and also for patients who need to switch inhaler.

Funding: Meda $\mathrm{AB}$.

Keywords: Asthma; Budesonide; Diskus ${ }^{\circledR}$; Dry powder inhaler (DPI); Easyhaler ${ }^{\circledR}$; Inhaler; Novolizer ${ }^{\circledR}$; Observational study; Patient preference; Turbuhaler ${ }^{\circledR}$

\section{INTRODUCTION}

Asthma is a common, chronic disease affecting around 300 million individuals worldwide [1]. Treatment of asthma with inhaled corticosteroids (ICSs) is recommended by the Global Initiative for Asthma (GINA) [1]. It is very important that the ICS is correctly taken [2]; however, poor compliance and incorrect handling of inhalers are frequent among people with asthma [3]. Patient preferences and opinions of the inhaler properties play a pivotal role for treatment outcomes. How patients perceive the handling of the device is often neglected [4]. Faulty technique can sabotage even the best of treatment plans for asthma [5].

Most patients cannot use the pressurized metered dose inhaler correctly [6, 7], and while dry powder inhalers (DPIs) are easier to use, patients also make mistakes when using these [8]. The Novolizer ${ }^{\circledR}$ (Meda, Sweden) inhaler device was specifically designed to overcome many of the disadvantages associated with other DPIs. Compared to other DPIs, the Novolizer has a low-medium intrinsic resistance, and still patients need only to generate an inspiratory flow rate of $35 \mathrm{~L} / \mathrm{min}$ for optimal drug delivery [9]. The dynamic resistance of the Novolizer is 5.5-times lower than that of the Turbuhaler ${ }^{\circledR}$ (AstraZeneca) and patients achieve significantly higher peak inspiratory flow rates and better inhalation performance with the Novolizer [10, 11]. The Novopulmon Novolizer deposits significantly more budesonide into the lungs than the Turbuhaler, more reliably, and with less variability of lung deposition [12-14]. In addition, the Novolizer is simple to use and simple to refill, rendering it forgiving of poor patient technique [15]. The optical, acoustic, and sensory feedback systems guide patients through a successful inhalation maneuver and provide confidence that the drug has reached the lungs [15-17].

The present study was performed in patients who were prescribed a switch of DPI in standard clinical practice by their physician. The main objective of this study was to determine the proportion of patients preferring the new inhaler upon switching device as well as after using new inhaler device. Secondary objectives were to determine the reasons for switching and to survey the patients' positive/negative opinions of different properties and functions of their ICS inhaler.

\section{METHODS}

\section{Patients}

Male and female asthma patients of $\geq 6$ years of age were included. To be eligible for the study, patients had to be receiving maintenance treatment with an ICS DPI (Step 2 according to GINA guidelines) [1] and a switch from the existing DPI to any other type of DPI was considered necessary. 


\section{Design}

This was a multicenter, prospective, observational study conducted at 50 primary care and hospital care centers in Sweden. The study was performed according to European regulations for non-interventional, observational studies [18, 19] and approved by the Ethics Committee of Lund, Sweden. All procedures followed were in accordance with the ethical standards of the responsible committee on human experimentation (institutional and national) and with the Helsinki Declaration of 1964, as revised in 2013. Informed consent was obtained from all patients for being included in the study. For patients below 18 years of age, both the patient and the patient's guardian received verbal and written information about the study and signed informed consent. The informed consent for patients 6-12 years of age was in a lay language intended for this group. The study period was carried out between January 2011 and September 2012. The new inhaler was prescribed by the investigator according to the summary of product characteristics (SPC). Patients with any contraindication according to the SPC to the new DPI treatment were excluded. For the switch, no study-specific procedure for informing and/or presenting the new inhaler was given. The intention was to keep the real-life procedures information/ demonstration intact in order to minimize influence on the results.

The study design incorporated a first visit together with a patient questionnaire concerning the previously used inhaler and a follow-up questionnaire with reference to different aspects of the new and old inhalers (function and properties) completed 2 months later.

\section{Data Collection}

The questionnaire was presented and collected by either a nurse or the investigator, in-line with routines at the specific clinic. A few questions were specific to the previous inhaler and the new inhaler had one unique question, which was preference for future use. Patients were asked to answer questions regarding appearance, handling, instruction for use, dose control, and global perception of the inhaler at study entry and at the second visit after 6-10 weeks. At the follow-up visit, a question regarding patient's preference of inhaler was asked. The novel questionnaire was based on pre-study discussions with experienced pediatrician/allergologist regarding the clinically relevant, key questions (Table S1 in the electronic supplementary material).

\section{Primary and Secondary Efficacy Endpoints}

The primary efficacy endpoint was the proportion of patients preferring the new inhaler. Secondary efficacy endpoints were patient's opinion of properties and functions of the inhaler both before and after the switch, past and present type of inhaler and the main reason for the patient's switch of inhaler.

\section{Statistics}

It was estimated that a sample size of 323 evaluable patients was required to obtain a two-sided 95\% confidence interval (CI) with the width $\pm 5 \%$ units for an expected proportion of patients who prefer the new inhaler of $70 \%$. The results were to be presented by age group (6-12 years, $13-17$ years, and $18+$ years). The primary efficacy endpoint, proportion of patients preferring the new inhaler was presented by a 
point estimate and a 95\% CI. Since the normal approximation fails when the proportion is close to 1 , an exact Clopper-Pearson CI was calculated.

The secondary endpoints were assessed on a 5-point ordinal scale (e.g., from very difficult to very easy) in 10 questions. Each of these endpoints was tested with a proportional odds model for repeated measure within patient. The factors age group, sex, status (old or new inhaler), and type of old inhaler were tested for inclusion in the model and the final model included significant factors only. The odds ratio (OR) with corresponding 95\% CI for new versus old inhaler and $P$ value are presented. In this study, an OR $>1$ implies that the new inhaler has a more favorable result (e.g., more easy to use). Answers to further four questions regarding functions of the inhaler and training on inhalers were presented as summary statistics. All statistical analyses were performed using SAS ${ }^{\circledR}$ (Version 9.3, SAS Institute Inc., Cary, NC, USA).

\section{Safety}

Suspected adverse drug reactions (ADRs) were assessed and documented in the case report form (CRF). The physician was required to document whether a reasonable causal relationship with the drug (yes/no) could be assigned for each adverse reaction reported.

\section{RESULTS}

\section{Patient Disposition}

The planned sample size of 323 patients was not reached at study termination after 19 months. After termination, a total of 280 subjects were recruited and of these 17 did not return for the final visit. Full patient disposition is provided in Table 1.

Since most patients switched from an ICS DPI to the Novopulmon Novolizer inhaler (98\%), a modified intent-to-treat (ITT) data set of these patients was selected for the efficacy evaluation. Very few patients switched to Diskus $^{\circledR} \quad$ (GlaxoSmithKline); 3 patients or Easyhaler $^{\circledR}$ (Orion); 2 patients and thus these were not included in the efficacy analysis (Table 1). To allow for comparison between the major brands, further 11 patients that had not used Diskus, Easyhaler, or Turbuhaler as the

Table 1 Patient disposition

\begin{tabular}{|c|c|c|c|c|}
\hline & Diskus & Easyhaler & $\begin{array}{l}\text { Novopulmon } \\
\text { Novolizer }\end{array}$ & Total \\
\hline Patients enrolled and allocated to treatment, $n(\%)$ & $3(1 \%)$ & $2(1 \%)$ & $275(98 \%)$ & 280 \\
\hline Patients excluded, $n$ & & & & 22 \\
\hline Did not return for follow-up & & & & 17 \\
\hline Protocol violations & & & & 3 \\
\hline Incomplete data & & & & 2 \\
\hline Number of subjects in safety analysis, $n(\%)$ & 3 & 2 & $253(92 \%)$ & $258(92 \%)$ \\
\hline Number of subjects in the modified ITT efficacy analysis ${ }^{\mathrm{a}}, n(\%)$ & 0 & 0 & $242(88 \%)$ & $242(86 \%)$ \\
\hline
\end{tabular}


Table 2 Patient demographics by age group, and sex: analysis data set

\begin{tabular}{lllll}
\hline & 6-12 years & 13-17 years & 18+ years & Total \\
\hline$n$ & 124 & 24 & 110 & 258 \\
Age (years) & & & & \\
Mean (SD) & $8.7(2.0)$ & $14.2(1.1)$ & $53.9(15.2)$ & $28.5(24.2)$ \\
Q1, Q3 & $7.0,10.0$ & $13.0,14.5$ & $41.0,67.0$ & $9.0,49.0$ \\
Median (min, max) & $8.0(6,12)$ & $14.0(13,17)$ & $55.5(19,85)$ & $13.0(6,85)$ \\
Sex & & & $37(34 \%)$ & $136(53 \%)$ \\
Male, $n$ (\%) & $86(69 \%)$ & $13(54 \%)$ & $73(66 \%)$ & $122(47 \%)$ \\
Female, $n(\%)$ & $38(31 \%)$ & $11(46 \%)$ & & \\
\hline
\end{tabular}

old ICS DPI were also excluded from the modified ITT efficacy analysis $(n=242)$.

\section{Patient Demographics}

The age and sex distribution of patients included in the analysis is shown in Tables 2 and 3 for the modified ITT efficacy analysis data set. The age of the participating patients ranged between 6 and 85 years.

\section{Previous Inhaler Use}

Turbuhaler, Diskus, and Easyhaler were the most commonly previously used DPIs for all age groups (Table 3). Nine patients had used other inhalators (including spray devices), of which four patients had previously used the DPI product Asmanex ${ }^{\circledR}$ (mometasone; Merck Sharp \& Dohme). The mean duration of use of previous inhaler was between 0.89 and 5.11 years (mean 4.50 , median 2.11 ).

Table 3 Patient demographics by age group, sex, and inhaler use: modified intent-to-treat efficacy analysis data set

\begin{tabular}{|c|c|c|c|c|}
\hline \multirow[t]{2}{*}{ Age group } & \multicolumn{3}{|c|}{ Old inhaler } & \multirow{2}{*}{$\begin{array}{l}\text { Novopulmon Novolizer } \\
\text { (new inhaler) }\end{array}$} \\
\hline & Diskus & Easyhaler & Turbuhaler & \\
\hline $6-12$ years & $30(25 \%)$ & $12(10 \%)$ & $76(64 \%)$ & $118(100 \%)$ \\
\hline Male & $24(80 \%)$ & $9(75 \%)$ & $51(67 \%)$ & $84(71 \%)$ \\
\hline Female & $6(20 \%)$ & $3(25 \%)$ & $25(33 \%)$ & $34(29 \%)$ \\
\hline $13-17$ years & $6(26 \%)$ & $1(4 \%)$ & $16(70 \%)$ & $23(100 \%)$ \\
\hline Male & $5(83 \%)$ & $0(0 \%)$ & $7(44 \%)$ & $12(52 \%)$ \\
\hline Female & $1(17 \%)$ & $1(100 \%)$ & $9(56 \%)$ & $11(48 \%)$ \\
\hline $18+$ years & $11(11 \%)$ & $11(11 \%)$ & $79(78 \%)$ & $101(100 \%)$ \\
\hline Male & $5(46 \%)$ & $5(46 \%)$ & $25(32 \%)$ & $35(35 \%)$ \\
\hline Female & $6(54 \%)$ & $6(54 \%)$ & $54(68 \%)$ & $66(65 \%)$ \\
\hline
\end{tabular}

Data are presented as $n(\%)$ 


\section{Efficacy}

\section{Primary Endpoint}

Almost all patients (98\%) were switched to the Novopulmon Novolizer as their new inhaler. For the primary outcome, the majority of patients selected the Novolizer as their choice of inhaler to continue using in the future: $92.4 \%$ (95\% CI 86.0-96.5\%) of 6-12-year-old, 90.9\% (95\% CI 70.8-98.9\%) of 13-17-year-old, and 83.3\% (95\% CI 74.4-90.2\%) of those aged 18 years and above.

\section{Secondary Endpoints}

Reason for Switching The main reasons for switching DPI (whether it is Diskus, Easyhaler, or Turbuhaler) were 'Insufficient asthma control' and 'Patient's wish' across all age groups, while handling problems were also frequently reported among those aged 6-12 years (Fig. 1). Insufficient asthma control appeared particularly pertinent to patients' desire to switch from the Easyhaler for all the age groups assessed. The main reasons given were not based on pre-defined definitions.

Inhaler Properties For all properties and functions, more outcomes are favorable for the Novopulmon Novolizer as compared to the previously used inhaler (Fig. 2). Compared with previously used inhalers, patients switched to the Novopulmon Novolizer found it easier to load, easier to inhale through, and easier to tell if they had inhaled the medicine. The patient's overall opinion of the Novopulmon Novolizer was also higher than that of previously used devices (OR 7.74; 95\% CI 5.27-11.4; $\quad P<0.0001)$. Compared with previously used inhalers, patients switched to Novopulmon Novolizer also found it easier to use, easy to learn how to use and easy to know how much drug was left in the inhaler. Patients preferred the shape and size of the Novopulmon
Novolizer compared with their previously used DPI and found it easier to carry with them (Fig. 2). Overall, the Novopulmon Novolizer was evaluated by patients as superior compared with the previously used devices ( $P<0.022$ for all comparisons).

Answers to four questions concerned with inhaler function and training on inhaler usage are presented as summary statistics. For all age groups, the most common ways in which patients learned to use their inhaler device (both old and new inhalers) was by oral presentation, physician demonstration, and third, nurse demonstration, with no
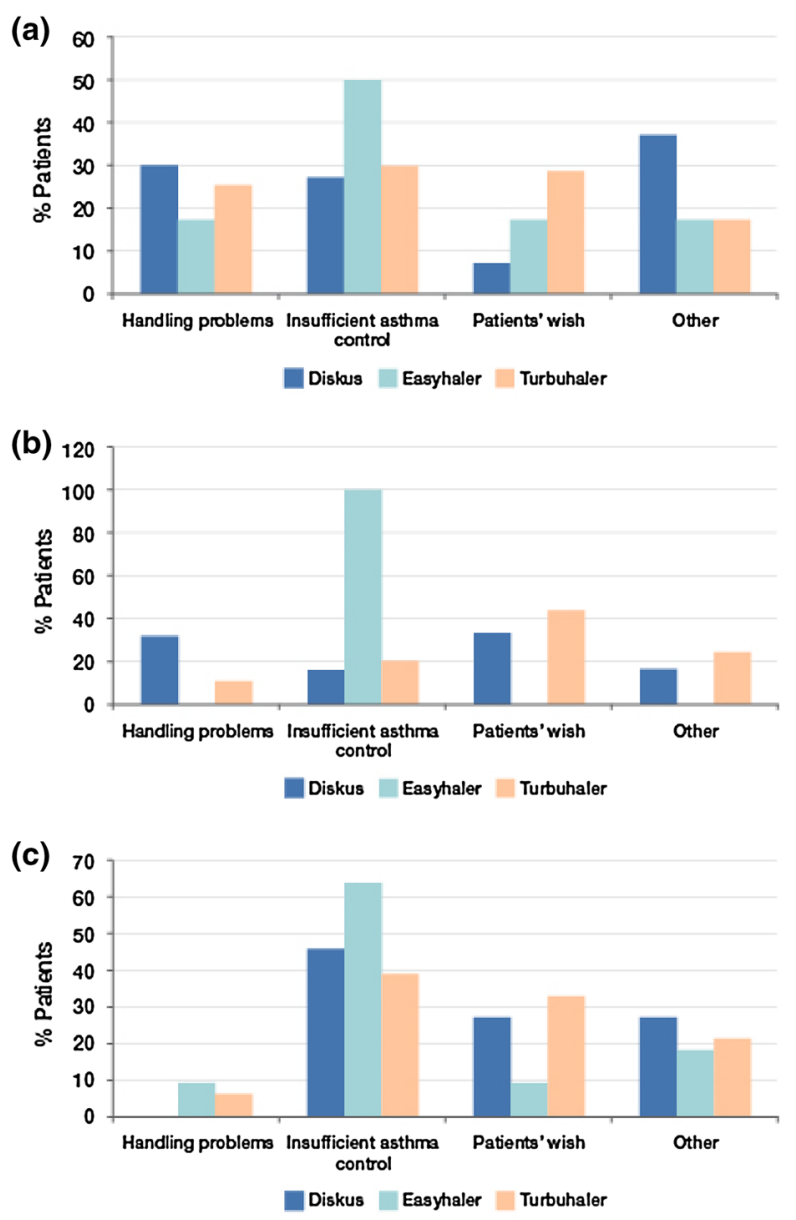

Fig. 1 Main reason for the patient's switch of inhaler, by type of old inhaler. a Age group 6-12 years; $\mathbf{b}$ age group $13-17$ years; $\mathbf{c}$ age group $18+$ years 


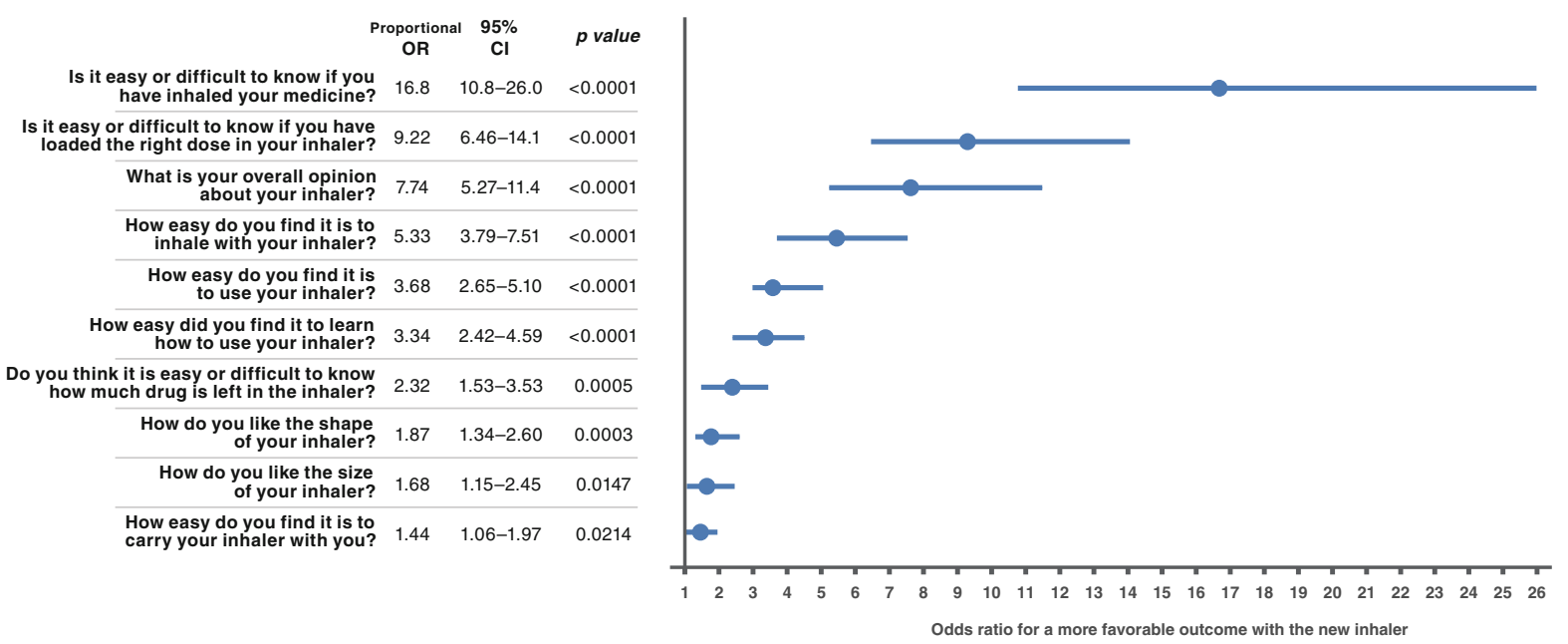

Fig. 2 Likelihood of a more favorable outcome with the new inhaler Novopulmon Novolizer compared with the previously used inhaler for answers given by patients to ten questions included in the questionnaire. The odds ratio

appreciable difference in teaching approach between the old and new device. Demonstration of inhaler technique by the patient occurred more frequently in the younger age groups (approximately 80\% of patients) than for those aged 18 years and above (approximately 50\% of patients).

Around one-fifth to one-half of all patients had received repeat information on how to use the old inhaler device prior to switching (18\% to 53\% across the age groups). Repeat demonstration of inhaler technique by the patient using the old device occurred more frequently among younger patients (58\% of $6-12$ year olds and $56 \%$ of 13-17 year olds) than among adults (18\%).

Patients judged that the most valuable functions for knowing if the medicine was taken were 'hearing a sound,' 'change of color,' and 'taste sensation.'

\section{Safety}

Three ADRs were reported in the study by patients that switched to the new inhaler
(OR) with corresponding $95 \%$ confidence interval (CI) for new versus old inhaler and $P$ value are presented; an OR $>1$ implies that the new inhaler has a more favorable result (e.g., more easy to use)

Novopulmon Novolizer, which led to treatment discontinuation; these included teeth discoloration, erythema, and cough.

\section{DISCUSSION}

This study was performed in patients who were prescribed a switch of DPI in standard clinical practice by their physician, and was designed to collect data on how patients evaluate a new inhaler when switching from a previously used inhaler. The key finding of this study is that the vast majority of patients across the age groups preferred to continue to use the new Novopulmon Novolizer at study end. A further finding of the study is that patients rated the new Novopulmon Novolizer inhaler as significantly superior versus the previous inhaler used across a number of questions concerned with properties and function of the inhaler device $(P<0.022$ for all comparisons). Compared with previously used inhalers, patients switched to Novopulmon Novolizer 
found it easier to use, easy to load and inhale through, and easy to know how much drug was left in the inhaler. The device attributes associated with patient preference for the Novopulmon Novolizer shown here, are consistent with recent findings by Price et al. [20]. They found higher device satisfaction with attributes including consistency in the amount of drug delivery to the lungs, ease of use, and feedback about the number of remaining doses. Higher patient satisfaction with their asthma drug delivery device was further shown to be a significant predictor of higher medication adherence and more favorable clinical outcomes [20]. The patient's preferences and opinions of the properties of the inhaler plays a pivotal role for treatment outcome, and the patient's perception of the handling of the device is often neglected [4]. However, the level of patient satisfaction with their inhaler device is demonstrated to have a positive influence on compliance and achievement of treatment goals for asthma [20, 21]. Regrettably, poor compliance and incorrect handling of inhalers is common among people with asthma [3, 22]. When technique is markedly flawed, suboptimal outcomes typically result, such as poor asthma control and increased frequency of emergency department visits [1, 5, 6, 22-24]. The Novolizer is a technically advanced DPI, which comprises a number of features that should improve compliance, safety, and efficacy. We have demonstrated that the Novolizer is associated with high patient preference, and it is suitable for patients with asthma regardless of severity [25].

The ease of handling of the Novopulmon Novolizer device with audible, visual, and sensory feedback mechanisms are attributes that contribute to the higher degree of patient preference. These attributes combined with medium airflow resistance makes the device suitable for use especially in very young patients able to use a DPI and for elderly patients who may not be capable of generating sufficient inhalation flow [16]. The Novolizer device has shown lower variability in lung deposition in vivo compared with the Turbuhaler device [14]. Handling problems with the previously used device were more frequently reported among the younger age group (aged 6-12 years) as the reason for switching. Future studies should explore the clinicians' and patient/guardians' views on switching from metered dose inhalers to DPIs in young patients suitable for DPIs.

Strength of this study is that it is a real-life clinical situation comparing previous inhaler use with the Novopulmon Novolizer. Randomized controlled trials usually exclude patients with suboptimal inhaler technique [3, 22]. Other published studies, systematic reviews, and guidelines have shown that patients do not get the full value of their inhaled medications because they use their inhaler incorrectly. Errors are made in inhalation technique and handling of the inhaler devices $[6,8,22,26]$.

The findings of our study are in-line with those of Perpiñá Tordera et al. [27] who investigated patient satisfaction and preference for three different inhalers, Novolizer, Turbuhaler, and Accuhaler after one week of use. In the younger age group below 16 years, the preferred inhaler was Novolizer (60\%) while Turbuhaler and Accuhaler were only preferred by $20 \%$ of patients in each case $(P=0.04)$. However, for the overall group no significant difference for preference was seen between the inhalers. In an earlier 4-week observational study, Novolizer was rated as better than previously used inhalers by $83 \%$ of patients [28]. In a further 4-week observational study, an improvement in compliance due to the control 
mechanisms of the Novopulmon Novolizer was observed in $80 \%$ of the 3057 patients [29].

At the time when the study started Novopulmon Novolizer had recently been launched in Sweden and was perceived among physicians as an inhaler with several unique features. This may have contributed to the high percentile switch to Novopulmon Novolizer (98\%). Another possible reason for the high preference for Novopulmon Novolizer could have been that the patients were discontent with their current inhaler at the time of switch. A high proportion of patients, $50-80 \%$, were able to demonstrate their inhalation technique with the previously used inhaler at the patient consultation and had used the old device for 0.89 to 5.11 years, but still a majority preferred the new inhaler at study end. The new inhaler Novopulmon Novolizer was well tolerated with few ADRs $(n=3) \quad$ reported that led to treatment discontinuation.

A limitation of the study is that it is not a randomized cross-over study where all inhalers are compared. Another possible limitation may be that patient expectation plays a role in perceived benefit. Clerisme-Beaty and co-workers [30] suggest that the methods by which treatment options are introduced to patients not only affect adherence to therapy and clinical outcomes, but also affect self-reported outcomes. The interaction between outcome expectancy and inhaler/drug efficacy is complex and includes that failure to notice the expected benefit may negatively affect adherence [30]. The level of satisfaction and preference for the Novopulmon Novolizer could have been influenced by a positive response to the novelty of the Novopulmon Novolizer. Nonetheless, the outcome was favorable for all parameters and should therefore also reflect a real-life advantage.

\section{CONCLUSIONS}

The main finding of this observational real-life study is that the vast majority of asthma patients (83.3\% to $92.4 \%)$ across all age groups preferred to use the new Novopulmon Novolizer inhaler compared with their previous inhaler devices (Turbuhaler, Diskus, and Easyhaler) and to continue to use the Novolizer beyond the study end. Furthermore, patients rated the Novolizer inhaler as significantly superior versus the previously used inhaler across all questions concerned with properties and function of the inhaler device $(P<0.022$ for all comparisons). In particular, the Novolizer's audible, visual and sensory feedback mechanisms (that identify whether the correct dose is loaded and administered) and its general ease of use were rated highly by asthma patients. These results are in-line with earlier study results in asthma and chronic obstructive pulmonary disease patients where the unique technical features of the Novolizer demonstrated significant advantages compared with other inhalers. Combined, this makes the Novopulmon Novolizer an ideal first choice inhaler for the treatment of asthma, also in patients who need to switch inhaler.

\section{ACKNOWLEDGMENTS}

All named authors meet the International Committee of Medical Journal Editors (ICMJE) criteria for authorship for this manuscript, take responsibility for the integrity of the work as a whole, and have given final approval to the version to be published. Sören Wille contributed to the conception and design of the study and was mainly responsible for overall recruitment of patients. Sören Wille and Holger 
von Fircks were responsible for interpretation of the data and for finalizing of the manuscript. All authors contributed to the intellectual content. The authors are grateful to all the patients in the study as well as the participating medical doctors and nurses at the clinics for their assistance in patient selection and data collection. We thank Olof Karlsson, Britta Lewald, Hélene Toll and Anne Bergman for their advice and contribution to the conception and design and Kerstin Wiklund for her contribution with the statistics. We also thank Olof Karlsson for his contribution to the manuscript. Sponsorship for this study and article processing charges was funded by Meda $\mathrm{AB}$. Editorial assistance in the preparation of this manuscript was provided by Lisa Buttle, PhD of MedScript Ltd. Support for this assistance was funded by Meda AB.

Disclosures. Sören Wille has received funding for lectures for Meda independently of this study. Holger von Fircks was Medical Advisor for the company Meda at the time of his contribution.

\section{Compliance with ethics guidelines. All} procedures followed were in accordance with the ethical standards of the responsible committee on human experimentation (institutional and national) and with the Helsinki Declaration of 1975, as revised in 2000 and 2008. The European regulations imposed for this non-interventional, observational study did not interfere with the physician's common therapy.

Open Access. This article is distributed under the terms of the Creative Commons Attribution-NonCommercial 4.0 International License (http://creativecommons.org/licenses/ by-nc/4.0/), which permits any noncommercial use, distribution, and reproduction in any medium, provided you give appropriate credit to the original author(s) and the source, provide a link to the Creative Commons license, and indicate if changes were made.

\section{REFERENCES}

1. Global Initiative for Asthma (GINA). The global strategy for asthma management and prevention. Updated 2014. http://www.ginasthma.org. Accessed 20 Aug 2015.

2. Levy ML, Hardwell A, McKnight, Holmes J. Asthma patients' inability to use a pressurised metered-dose inhaler (pMDI) correctly correlates with poor asthma control as defined by the Global Initiative for Asthma (GINA) strategy: a retrospective analysis. Prim Care Respir J. 2013;22:406-11.

3. Molimard M, Raherison C, Lignot S, Depont F, Abouelfath A, Moore N. Assessment of handling of inhaler devices in real life: an observational study in 3811 patients in primary care. J Aerosol Med. 2003;16:249-54.

4. Mäkelä MJ, Backer V, Hedegaard M, Larsson K. Adherence to inhaled therapies, health outcomes and costs in patients with asthma and COPD. Respir Med. 2013;107:1481-90.

5. Self TH, Wallace JL, George CM, Howard-Thomson A, Schrock SD. Inhalation therapy: help patients avoid these mistakes. J Fam Pract. 2011;60:714-21.

6. Cochrane MG, Bala MV, Downs KE, Mauskopf J, Ben-Joseph RH. Inhaled corticosteroids for asthma therapy: patient compliance, devices, and inhalation technique. Chest. 2000;117:542-50.

7. Crompton GK. Problems patients have sing pressurized aerosol inhalers. Eur J Respir Dis. 1982;63(Suppl 119):101-4.

8. Lavorini F, Magnan A, Dubus JC, et al. Effect of incorrect use of dry powder inhalers on management of patients with asthma and COPD. Respir Med. 2008;102:593-604.

9. Berner B, Fyrnys B, deBoer AN, et al. ASTA Medica multi dose dry powder inhaler. In: Dalby RN, Byron PR, Farr SJ, editors. Respiratory drug delivery VI. Buffalo Grove: Interpharm Press; 1998. p. 475-7.

10. Cegla UH. Pressure and inspiratory flow characteristics of dry powder inhalers. Respir Med. 2004;98(Suppl A):S22-8. 
11. Richter K. Successful use of DPI systems in asthmatic patients-key parameters. Respir Med. 2004;98(Suppl B):S22-7.

12. Newman SP, Pitcairn GR, Hirst PH, et al. Scintigraphic comparison of budesonide deposition from two dry powder inhalers. Eur Respir J. 2000;16(1):178-83.

13. Newman SP. Drug delivery to the lungs from dry powder inhalers. Curr Opin Pulm Med. 2003;9(Suppl 1):S17-20.

14. Munzel U, Marschall K, Fyrnys B, Wedel M. Variability of fine particle dose and lung deposition of budesonide delivered through two multidose dry powder inhalers. Curr Med Res Opin. 2005;21:827-33.

15. Kohler D. Novolizer: the new technology for the management of asthma therapy. Curr Opin Pulm Med. 2003;9(Suppl 1):S11-6.

16. Fyrnys B, Stang N, Wolf-Heuss E. Stability and performance characteristics of a budesonide powder for inhalation with a novel dry powder inhaler device. Curr Opin Pulm Med. 2001;7(Suppl):S7-11.

17. Siddiqui MA, Plosker GL. The Novolizer: a multidose dry powder inhaler. Treat Respir Med. 2005;4(1):63-9.

18. European Union Directive 2001/20/EC of the European Parliament and of the Council of 4 April 2001 on the approximation of the laws, regulations and administrative provisions of the member States relating to the implementation of good clinical practice in the conduct of clinical trials on medicinal products for human use. Off J Eur Communities 2001;L 121:34-44.

19. R6ICH E2E: Pharmacovigilance planning. Note for guidance on planning pharmacovigilance activities $\mathrm{CPMP} / \mathrm{ICH} / 5716 / 03$ (EU Step 5) London, 1 December 2004, valid as of June 2005.

20. Price D, Harrow B, Small M, Pike J, Higgins V. Establishing the relationship of inhaler satisfaction, treatment adherence, and patient outcomes: a prospective, real-world, cross-sectional survey of US adult asthma patients and physicians. World Allergy Organ J. 2015;8(1):26. doi:10.1186/s40413015-0075-y.
21. Small M, Anderson P, Vickers A, Kay S, Ferner S. Importance of inhaler-device satisfaction in asthma treatment: real-world observations of physician-observed compliance and clinical/patient-reported outcomes. Adv Ther. 2011;28(3):202-12.

22. Haughney J, Price D, Barnes NC, Virchow JC, Roche $\mathrm{N}$, Christyn H. Choosing inhaler devices for people with asthma: current knowledge and outstanding research needs. Respir Med. 2010;104:1237-45.

23. Al-Jahdali $\mathrm{H}$, Ahmed A, Al-Harbi A, et al. Improper inhaler technique is associated with poor asthma control and frequent emergency department visits. Allergy Asthma Clin Immunol. 2013;9:8.

24. Brocklebank D, Ram F, Wright J, et al. Comparison of the effectiveness of inhaler devices in asthma and chronic obstructive airways disease: a systematic review of the literature. Health Technol Assess. 2001;5:1-149.

25. Virchow JC. What plays a role in the choice of inhaler device for asthma therapy? Curr Med Res Opin. 2005;21(Suppl 4):S19-25.

26. Lenney J, Innes JA, Crompton GK. Inappropriate inhaler use: assessment of use and patient preference of seven inhalation devices. EDICI. Respir Med. 2000;94:496-500.

27. Tordera MP, Viejo JL, et al. Assessment of patient satisfaction and preferences with inhalers in asthma with the FSI-10 Questionnaire. Arch Bronconeumol. 2008;44:346-52.

28. Möller M, Grimmbacher S, Munzel U. Improvement of asthma therapy by a novel formoterol multidose dry powder inhaler. Arzneimittel-Forschung (Drug Res). 2008;58:168-73.

29. Möller $M$, Fritsche D, Rivera D, Libertus $H$. Improvement of asthma therapy by a novel budesonide multidose dry powder inhaler. Arzneimittel-Forschung (Drug Res). 2003;53:562-7.

30. Clerisme-Beaty EM, Bartlett SJ, Teague WG, et al. The Madison Avenue effect: how drug presentation style influences adherence and outcome in patients with asthma. J Allergy Clin Immunol. 2011;127(2):406-11. 